\title{
Flow Cytometry Crossmatch and Kidney Graft Outcome
}

\author{
A. Piazza, L. Borrelli, O. Buonomo, F. Pisani, M. Valeri, N. Torlone, A. Felici, P.I. Monaco, D. Adorno, \\ and C.U. Casciani
}

$I^{\mathrm{T}}$ IS WELL accepted that flow cytometric detection before the renal transplant of donor-specific antibodies is associated with a high incidence of acute rejection (AR) episodes $^{1-4}$ and with decreased long-term graft survival., On the other hand, the clinical relevance of a positive flow cytometric crossmatch (FCXM) posttransplantation is not yet established.

FCXM has been shown to be more advantageous in the detection of anti-donor antibodies ${ }^{7}$ than standard complement-dependent lymphocytotoxic crossmatch (CDC); ${ }^{8}$ it allows the simultaneous analysis of complement-activating and nonactivating alloantibodies, the class of donor-specific antibodies (IgG and/or IgM), and the kind of donor target cells ( $\mathrm{T}$ and/or B lymphocytes). Therefore, posttransplant FCXM seems to be a good approach to monitor donorspecific immune response., ${ }^{4,9}$ In fact, as shown by our previous data, ${ }^{10}$ FCXM routinely performed after transplantation represents a specific, sensitive, and noninvasive method for monitoring AR in cadaveric renal transplants.

The aims of the present study were to verify in a wide kidney transplant (KT) patient population if posttransplant FCXM monitoring can be considered a sensitive method for the assessment of $\mathrm{AR}$ in renal transplantation and to demonstrate its ability in predicting graft failure.

\section{MATERIALS AND METHODS \\ Patients}

Sixty-six KT recipients, who received cadaveric KTs in the Transplant Unit of Clinical Surgery at Tor Vergata University of Rome between January 1995 and June 1997, were enrolled in the study. All patients were pretransplant FCXM negative. Organ allocation was based on the best donor-recipient HLA matching (matching priority: HLA-DR, HLA-B, and HLA-A).

FCXM monitoring was performed on sera samples taken from patients before and after transplantation on days 7, 14, 30, 60, 90, and when acute rejection (AR) was suspected. All sera samples were checked for donor-specific antibodies and autoantibodies using FCXM. Donor spleen lymphocytes were stored in liquid nitrogen until use.

Immunosuppressive therapy included cyclosporine (CyA), azathioprine, and prednisone. Rejection episodes were diagnosed by assessing clinical symptoms and were confirmed usually by needle core biopsy. Rejection treatment consisted of three boluses of methylprednisolone. Clinical follow-up data were collected for the first 12 months after transplantation.

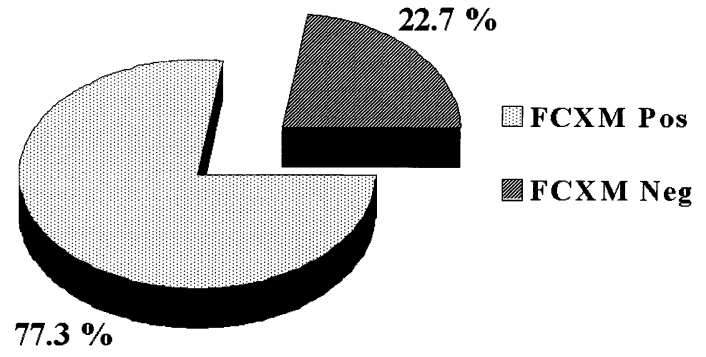

Fig 1. Posttransplant FCXM monitoring in 66 KT patients.

FCXM

Donor spleen lymphocytes $\left(2.5 \times 10^{5}\right)$ and serum $(75 \mu \mathrm{L})$ were incubated for 30 minutes at room temperature. After two wash steps with phosphate-buffered saline (PBS) containing 5\% fetal bovine serum and $0.1 \%$ of $\mathrm{NaN}_{3}$ (PBS flow), the lymphocytes were incubated for 30 minutes at $4^{\circ} \mathrm{C}$ with $50 \mu \mathrm{L}$ of fluorescein isothiocyanate-conjugated anti-human IgG or IgM F(ab')2 (1/50 diluted; Dako-Denmark) to determine the class of bound alloantibodies; 5 $\mu \mathrm{L}$ of peridinin chlorophyll protein (PerCP) anti-CD3 and $5 \mu \mathrm{L}$ of phycoerythrin (PE) anti-CD20 (Becton Dickinson, San Jose, Calif) were also added to the cell suspension to identify donor $\mathrm{T}$ and $\mathrm{B}$ lymphocytes. The cells were then washed twice with PBS flow and resuspended for fixation in $200 \mu \mathrm{L}$ of $1 \%$ paraformaldehyde in PBS until analysis.

For all FCXMs, a positive control serum (pool of patients' sera with $>90 \%$ panel reactive antibody [PRA]) and a negative control serum (pool of more than five sera samples from healthy male subjects) were also incubated with donor lymphocytes. Samples were analyzed using a FACScan flow cytometer and FACScan software (Becton-Dickinson). A channel shift of more than 2 SD between the mean log fluorescence of the negative control sample and test samples was considered to be a positive FCXM.

\section{Data Analysis}

The chi-square and Mann-Whitney two-sample tests were used for statistical comparison. Values of $P<.05$ were considered to be statistically significant.

From the CNR Institute of Tissue Typing, Unit of Rome, and Department of Surgery Tor Vergata University, Rome, Italy.

Supported in part by CNR Target Project on "Biotechnology." Address reprint requests to $\mathrm{Dr}$ D. Adorno, Istituto CNR di Tipizzazione Tisoutale, Sez. di Roma, c/o Ospedale S. Eugenio, Piazz le dell'Umanesimo 10, 00144 Roma, Italy. 

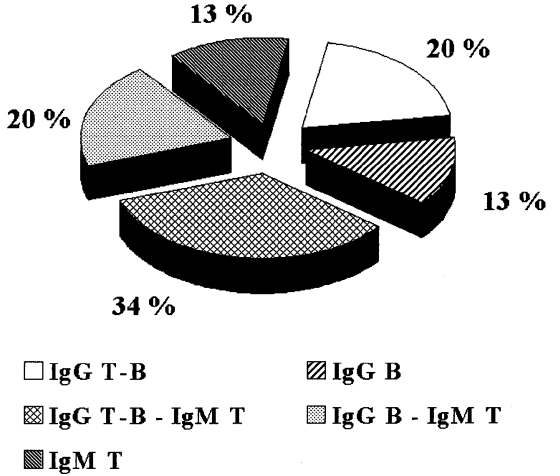

Fig 2. Class and specificity of alloantibody detected using the FCXM technique.

\section{RESULTS}

\section{FCXM Monitoring}

Fifteen of the $66(22.7 \%)$ KT recipients proved, at least, once FCXM positivity during the first 3 months after transplantation (Fig 1). As regard the class of alloantibodies and the type of target lymphocytes, the analysis of FCXM positivity showed (Fig 2) IgG anti-T and B lymphocytes in $20 \%$ (3 of 15) of patients; IgG only anti-B lymphocytes in $13 \%$ (2 of 15) of patients; IgG anti-T and B lymphocytes and IgM anti-T cells in 34\% (5 of 15) of patients; IgG anti-B lymphocytes and IgM anti-T cells in 20\% (3 of 15) of patients; and only IgM anti-T lymphocytes in $13 \%$ ( 2 of 15 ) of patients.

\section{HLA-A, B, DR Compatibility}

We found an increase, although it was not significant, in the degree of HLA-A, B mismatch in the FCXM-positive group compared with the FCXM-negative patients (HLA-A, B mismatch mean $\pm \mathrm{SD}: 3 \pm 0.60$ vs $2.25 \pm 0.88)$. On the other hand, no differences were observed in the degree of HLA-DR mismatching in the two groups (HLA-DR mismatch mean $\pm \mathrm{SD}: 0.74 \pm 0.62$ vs $0.56 \pm 0.48)$ (Table 1$)$.

\section{$\mathrm{AR}$ and FCXM}

Twenty-one AR episodes occurred in 17 patients; in all but 3 patients the first AR episodes appeared within 15 days after transplantation and 4 patients had a second rejection episode within the third month. Five grafts were lost during the first posttransplant year due to immunologic causes. Correlating AR and posttransplant FCXM monitoring, we

Table 1. Analysis of HLA-A, B, DR Mismatches According to FCXM Status

\begin{tabular}{lcc}
\hline & $\begin{array}{c}\text { FCXM Positive } \\
(\mathrm{n}=15)\end{array}$ & $\begin{array}{c}\text { FCXM } \\
\text { Negative } \\
(\mathrm{n}=51)\end{array}$ \\
\hline HLA-AB mismatches $^{*}$ & $3.00 \pm 0.60$ & $2.25 \pm 0.88$ \\
HLA-DR mismatches $^{*}$ & $0.74 \pm 0.62$ & $0.56 \pm 0.48$ \\
\hline
\end{tabular}

${ }^{*}$ Data are given as mean \pm SD.
Table 2. Correlation Between Posttransplant FCXM Monitoring and AR Incidence

\begin{tabular}{lcc}
\hline & AR $+(\%)$ & AR- (\%) \\
\hline FCXM+ & 80 & 20 \\
FCXM- & 9.8 & 90.2 \\
\hline
\end{tabular}

found that 12 of the $15(80 \%)$ FCXM-positive patients experienced at least one AR episode; all 4 patients who experienced two episodes of AR were in the FCXMpositive group. On the other hand, in the 51 FCXMnegative patients only $5(9.8 \%)$ had AR episodes $(P=$ .00002) (Table 2).

\section{Graft Function and FCXM}

Analyzing the correlation between the serum creatinine level at 3, 6, and 12 months posttransplant and the FCXM positivity, we observed that the mean serum creatinine value in FCXM-positive patients was significantly higher than that seen in FCXM-negative patients $(2.92 \pm 1.78$ vs $1.78 \pm$ $0.69 \mathrm{mg} / \mathrm{dL}, P=.0003$ at 3 months; $2.39 \pm 1.19$ vs $1.76 \pm 0.58$ $\mathrm{mg} / \mathrm{dL}, P=.0094$ at 6 months; $2.92 \pm 1.69$ vs $1.65 \pm 0.53$ $\mathrm{mg} / \mathrm{dL}, P=.0001$ at 1 year posttransplant) (Fig 3).

\section{Graft Outcome and FCXM}

Five KT patients presented graft failure within the first year posttransplantation. All these patients who lost their graft were both FCXM and AR positive (5 of $12=41.7 \%$ ). On the contrary, patients who were only FCXM positive or only AR positive did not lose the graft during the same posttransplant period $(P=.001)$ (Fig 4).

\section{DISCUSSION}

The presence before renal transplant of anti-donor antibodies detected by using CDC crossmatch represents an absolute contraindication to transplantation. Recently, a new flow cytometric crossmatch technique, more sensitive than the standard CDC crossmatch, ${ }^{1,11}$ has been used. By apply-

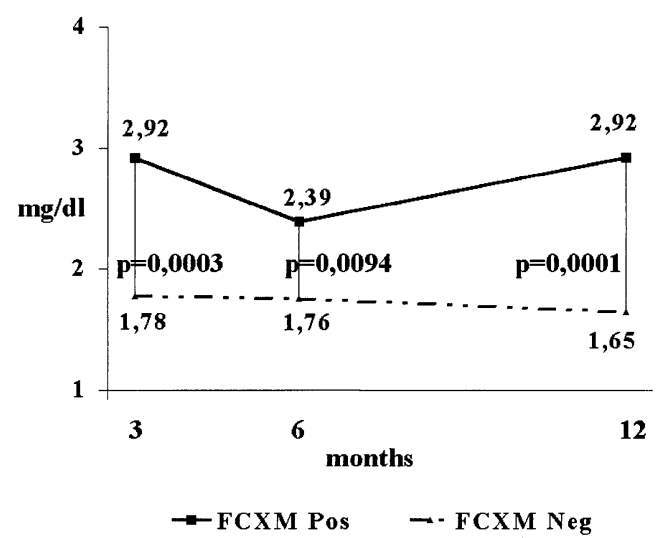

Fig 3. Graft function during the first year posttransplant in the FCXM-positive and negative group of patients. 


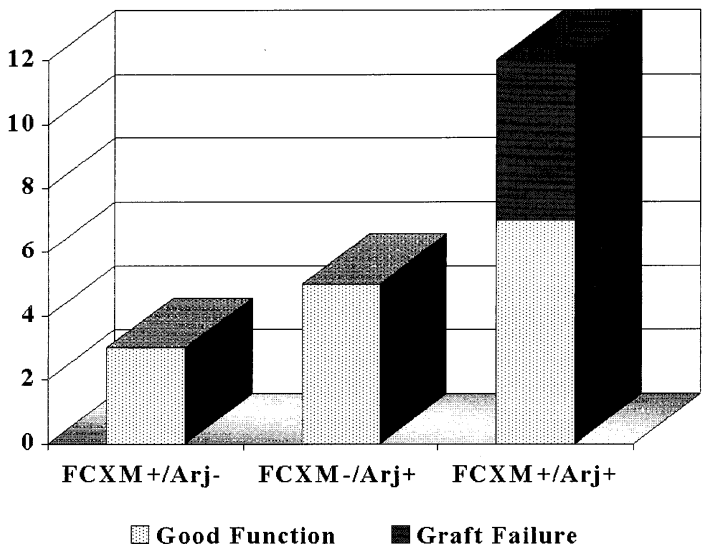

Fig 4. Analysis of graft outcome in relation to FCXM data and AR incidence.

ing this method, some authors described a higher incidence of rejection episodes in patients who had a positive FCXM before transplant. ${ }^{2,12}$ On the contrary, the importance of a positive FCXM after transplantation is still unclear.

In accordance with published data, ${ }^{4,10,13}$ our study on 66 KT patients has shown that the patients with a positive FCXM after transplantation had a statistically very significant higher incidence of AR than the FCXM-negative patients $(80 \%$ vs $9.8 \%, P=.00002)$. Analyzing graft function in the same two groups of patients during followup, we found a significantly poor graft function in the FCXM-positive patients.

By immunohistologic studies of biopsy performed during chronic rejection, the deposit of immunoglobulin and complement in areas of intimal thickening ${ }^{14}$ was observed and it has been postulated that the humoral immune response represents one of the most important factors that causes the development of chronic rejection and graft loss. All this correlates well with our observation of harmful effect of donor-specific antibodies to renal graft function.
In fact, in this study correlating FCXM monitoring to AR incidence, we have found that all five patients who lost the graft within the first year posttransplant were both FCXM positive and AR positive. On the bases of these data, we can hypothesize that the detection of posttransplant alloantibodies represents a negative prognostic factor for the clinical outcome of the renal graft.

In conclusion, our study suggests that FCXM is a sensitive noninvasive tool for the assessment of rejection in renal transplant recipients. It also suggests that FCXM may be a good method for predicting long-term graft outcome and for improving graft survival by adapting "specific" immunosuppressive treatment which may delay the graft loss.

\section{REFERENCES}

1. Garavoy MR, Rheinschmidt MA, Bigos M, et al: Transplant Proc 15:1939, 1983

2. Lazda VA, Pollak R, Mozes MF, et al: Transplantation 45:562, 1988

3. Thistlethwaite JR Jr, Buckingham MR, Stuart JK, et al: Transplant Proc 18:676, 1986

4. Scornik JC, Salomon DR, Lim PB, et al: Transplantation 47:287, 1989

5. Mahoney RJ, Ault KA, Given SR, et al: Transplantation 49:527, 1990

6. Chapman JR, Deierhoi MH, Carter NP, et al: Transplant Proc 17:2480, 1985

7. Sutton PM, Harmer AW, Bayne AM, et al: Transplant Int $8: 360,1995$

8. Terasaki PI, McClelland JD: Nature 204:998, 1964

9. Buchler M, Al Najjar A, Guerraoui A, et al: Transplant Proc 27:2478, 1995

10. Piazza A, Adorno D, Poggi E, et al: Transplant Proc 30:1769

11. Berteli AJ, Daniel V, Pomer S, et al: Transplant Proc 22:1895, 1990

12. Ogura K, Terasaki PI, Johnson C, et al: Transplantation 56:294, 1993

13. Utzig MJ, Blümke M, Wolff-Vorbeck G, et al: Transplantation 63:551, 1997

14. O’Connel TX, Mobray JF: Transplantation 51:262, 1973 